УДК 519.41/47

\title{
Shunkov Groups with the Minimal Condition for Noncomplemented Abelian Subgroups
}

\author{
Nikolai S. Chernikov* \\ Institute of Mathematics \\ National Academy of Sciences of Ukraine \\ Tereschenkivska, 3, Kyiv-4, 01601 \\ Ukraine
}

Received 10.09.2015, received in revised form 21.09.2015, accepted 02.11.2015

In the present paper, we give a complete exhaustive description of the pointed out Shunkov groups.

Keywords: Shunkov, periodic, locally finite, completely factorizable, Chernikov group, minimal conditions, complemented, abelian subgroups.

DOI: $10.17516 / 1997-1397-2015-8-4-377-384$

\section{Introduction}

A great many deep and bright results are connected with groups, satisfying various minimal conditions, and with groups, having wide systems of complemented subgroups (see, for instance, $[1-7])$.

The present paper is devoted to the Shunkov groups with the minimal condition above.

Below $p$ and $q$ are always primes; $\min -a b, \min -a b \bar{c}, \min -p$ and $\min -p^{\prime}$ are the minimal conditions respectively for abelian, abelian noncomplemented, for $p$ - and $p^{\prime}$-subgroups. All other notations are standard.

Remind that the group $G$ is called Shunkov, if for any its finite subgroup $K$, every subgroup of the factor group $N_{G}(K) / K$, generated by two conjugate elements of prime order, is finite (V.D. Mazurov, 1998). The class of Shunkov groups is wide and includes, for instance, binary finite groups, 2-groups. The known Suchkova-Shunkov Theorem [8] (see also [4, Theorem 4.5.1]) asserts: The Shunkov group with $\min -a b$ is Chernikov.

Further, remind that the subgroup $H$ of the group $G$ is called complemented in $G$, if for some subgroup $K$ of $G, G=H K$ and $H \cap K=1 ; K$ is called a complement of $H$ in $G$. The group $G$ is called completely factorizable, if every its subgroup is complemented in it (N. V. Chernikova [9]). The fundamental N. V. Chernikova's Theorem $[9,10]$ (see also, for instance, [1, Theorem 7.2]) gives an exhaustive description of completely factorizable groups and asserts: The group $G \neq 1$ is completely factorizable iff $G=A \lambda B$ where $A$ is a direct product of normal subgroups of prime orders of $G$ and $B$ is a direct product of subgroups of prime orders or $B=1$; in particular, the $p$-group $G$ is completely factorizable iff it is elementary abelian. The known Kargapolov [11]Gorchakov [12] Theorem asserts: The group is completely factorizable iff all its abelian subgroups are complemented.

\footnotetext{
*chern@imath.kiev.ua

(C) Siberian Federal University. All rights reserved
} 
It is natural to consider groups, having infinite abelian subgroups, in which all such subgroups are complemented. B. I. Mishchenko [13] has described the infinite solvable and the infinite radical in the sense of B. I. Plotkin groups with complemented infinite abelian subgroups (see Theorem 1 [13] and Corollary [13, p. 158]). Since all such groups are locally finite, it is natural to consider the locally finite groups with $\min -a b \bar{c}$. N. S. Chernikov $[14,15]$ has described these locally finite groups (see Theorem [15] and Corollary 3.5 [15]). N. S. Chernikov [14, 16] has established that binary finite groups with $\min -a b \bar{c}$ are locally finite (see Theorem 3 [16]).

\section{The main result and some corollaries}

The author succeeded in proving the following general theorem, which is the main result of the present paper.

Theorem. For the Shunkov group $G$ the following statements are equivalent:

(i) $G$ satisfies the minimal condition for abelian noncomplemented subgroups.

(ii) $G$ is a Chernikov group or a non-Chernikov group with complemented infinite abelian subgroups.

(iii) $G$ is a Chernikov group or $G$ is a completely factorizable group, or $G=A \lambda B$ where $A$ is infinite and $A$ is a direct product of normal in $G$ subgroups of prime orders, $B=C \times D$ is finite, $C$ is a direct product of subgroups of prime orders or $C=1, D$ is cyclic $\neq 1$ and for every $p \in \pi(D), p^{2}|| D \mid$, and also for every $g \in D \backslash\{1\}, C_{A}(g)$ is finite. finite.)

(In view of O.Yu. Shmidt's Theorem (see, for instance, [20, Theorem 1.45]), in (iii) $G$ is locally

Theorem is equivalent to the author's Theorem [17].

Theorem implies the following proposition.

Proposition ([17]). The Shunkov p-group $G$ (in particular, the 2-group G) satisfies the minimal condition for abelian noncomplemented subgroups iff it is Chernikov or elementary abelian.

Note that Theorem [17] and Proposition [17] are exactly all results of [17].

The following new author's assertions are the immediate consequences of Proposition.

Corollary 1. For the 2-group $G$ the following statements are equivalent:

(i) $G$ satisfies the minimal condition for abelian noncomplemented subgroups.

(ii) G satisfies the minimal condition for noncomplemented subgroups.

(iii) $G$ is Chernikov or elementary abelian.

Corollary 2. For the Shunkov p-group $G$ the following statements are equivalent:

(i) $G$ satisfies the minimal condition for abelian noncomplemented subgroups.

(ii) $G$ satisfies the minimal condition for noncomplemented subgroups.

(iii) $G$ is Chernikov or elementary abelian.

In connection with the results above, note that for every $p>665$, there exists the non-solvable group of exponent $p$ containing an infinite abelian subgroup, in which every abelian subgroup of order $>p$ is complemented (N.S. Chernikov [18]). Thus the above requirements: " $G$ is a 2-group", "G is Shunkov" are essential. 


\section{Proof of the main result}

A. Show that (i) implies (iii).

Let (i) hold. The subsequent proof will be accomplished in a series of steps.

(1) $G$ is periodic.

Proof. Let $G$ have some element $g$ of infinite order. Then some subgroup $<g^{2^{n}}>$ of the infinite chain $<g^{2}>\supset<g^{4}>\supset \ldots \supset<g^{2^{k}}>\supset<g^{2^{k+1}}>\supset \ldots$ has a complement $D$ in $G$. But $1<|D \cap<g\rangle \mid<\infty$, which is a contradiction.

(2) If $G$ has a normal infinite locally finite subgroup $H$, then the statement (iii) is valid.

Proof. First, let $H$ be Chernikov. Now remind the following S. N. Chernikov's Proposition (see, for instance, [1, Proposition 1.13, p. 62]): A periodic group of automorphisms of the group, which is a direct product of finitely many quasicyclic subgroups, is finite. Further, $H$ contains the characteristic subgroups $R$ of finite index, which is such product. Since $G$ is periodic (see (1)), in view of the last Proposition, $\left|G: C_{G}(R)\right|<\infty$. In accordance with Lemma 1.1 [15], an abelian group with $\min -a b \bar{c}$ is precisely Chernikov or a direct product of groups of prime orders. Every maximal abelian subgroup of $C_{G}(R)$ satisfies min $-a b \bar{c}$ and is not such product and so is Chernikov. Hence follows: $G$ satisfies $\min -a b$. Therefore in virtue of Suchkova-Shunkov Theorem [8] (see above), $G$ is Chernikov and, at the same time, (iii) is valid.

Now let $H$ be non-Chernikov. Remind the following N.S. Chernikov's Theorem (see [15, Theorem]): The locally finite group with $\min -a b \bar{c}$ is the same as in (iii). Consequently, with regard to N. V. Chernikova's Theorem (see, above), $H=K \lambda L$, where $K$ is a direct product of normal in $H$ subgroups of prime orders, $L$ is abelian without quasicyclic subgroups. Let $F$ be the Fitting subgroup of $H$. Then $F$ is locally nilpotent and $F=K \lambda(F \cap L) \unlhd G$. Since $H$ is solvable, in view of Proposition 5.4.4 (ii) [19, (see p. 144)], $C_{H}(F)=Z(F)$. Therefore because of $H$ is infinite, $F$ is infinite too. Obviously, $F$ is non-Chernikov. Further, every mentioned direct multiplier of $K$ belongs to $Z(F)$ (for instance, in view of Proposition 1.16 [1, (see p. 70)]). So $F$ is abelian. In accordance with Lemma 1.9 [15], the group, satisfying $\min -a b \bar{c}$ and having a normal abelian non-Chernikov subgroup, is the same as in (iii). Thus (iii) is valid.

(3) Either the statement (iii) is valid, or the product $L$ of all normal locally finite subgroups of $G$ is finite and also $G$ includes some normal infinite subgroup $M$, which does not satisfy min $-a b$ and has no subnormal locally finite subgroups $\neq 1$.

Proof. Assume that (iii) is not valid. Then $G$ is infinite. In consequence of O.Yu. Shmidt's Theorem (see, for instance, [20, Theorem 1.45]), $L$ is locally finite. By virtue of the assertion (2), $L$ is finite. So $\left|G: C_{G}(L)\right|<\infty$. Again by virtue of (2), $C_{G}(L)$ is not locally finite. Therefore, with regard to Suchkova-Shunkov Theorem [8] (see above), $C_{G}(L)$ does not satisfy $\min -a b$. So some maximal abelian subgroup $A$ of $C_{G}(L)$ is not Artinian. Clearly, $L \cap C_{G}(L) \subseteq Z\left(C_{G}(L)\right)$ and so $L \cap C_{G}(L) \subseteq A$. Further, $A$ has some infinite descending series

$$
A=A_{0} \supset A_{1} \supset A_{2} \supset \ldots \supseteq \cap_{n=1}^{\infty} A_{n} \supseteq L \cap C_{G}(L) \supseteq 1 .
$$

Some $A_{n}$ has a complement $D$ in $G$. Put $M=<(D \cap A)^{G}>$. In view of Chunikhin's Lemma (see, for instance, [21, Lemma 1.36]), $M \subseteq D$. Also $M \subseteq C_{G}(L)$ and $D \cap L \cap C_{G}(L)=1$. So $M \cap L \subseteq\left(D \cap C_{G}(L)\right) \cap L=1$. In consequence of Theorem 1.1 in $\S 2$ of Chapter 5 [22] (see [22, p. 345]), every subnormal locally finite subgroup of $M$ belongs to $L$. Consequently, $M$ has no subnormal locally nontrivial subgroups. Also with regard to Suchkova-Shunkov Theorem, $M$ does not satisfy $\min -a b$. 
(4) If $G$ is a p-group, then (iii) is valid and, at the same time, $G$ is Chernikov or elementary abelian.

Proof. Let $G$ be a $p$-group. It is easy to see, with regard to N.V. Chernikova's Theorem above: $G$ is Chernikov or elementary abelian iff (iii) is valid.

Assume that (iii) is not valid. Now define the finite subgroup $H$ of $G$ in the following way.

First, if $G$ has an element $g$ of order $p^{2}$, then put $H=\langle g\rangle$.

Suppose that $G$ is of exponent $p$. Then $G$ is non-abelian. If for some $g, h \in G,\left[g, g^{h}\right] \neq 1$, then we put $H=<g, g^{h}>$. Since $G$ is Shunkov, $H$ is finite. Further, assume that also for every $g \in G$ and $h \in G,\left[g, g^{h}\right]=1$. Take $a, b \in G$ such that $[a, b] \neq 1$. Since $<a^{h}: h \in G>$ and $<b^{h}: h \in G>$ are normal abelian subgroups of $G$, the subgroup $<a^{h}: h \in G><b^{h}: h \in G>$ is metabelian and non-abelian. Further, the known S. N. Chernikov's Theorem (see, for instance, [1, Proposition 1.1]) asserts: Periodic locally solvable groups are locally finite. Then $\langle a, b\rangle$ is finite non-abelian. Now put $H=<a, b>$.

Let $A$ be any abelian subgroup of $C_{G}(H)$. Then $A H$ is a nilpotent non-(elementary abelian) group with $\min -a b \bar{c}$. In accordance with Lemmas 2.2 [15] and 1.1 [15]: Every non-Chernikov locally nilpotent $p$-group with min $-a b \bar{c}$ is elementary abelian. Thus, $A H$ is Chernikov. So $C_{G}(H)$ satisfies $\min -a b$. Now remind Shunkov's Theorem [23]: The 2-group with min - $a b$ is Chernikov. Remind An. Ostilovskiy's Theorem [24] (see also [4, Theorem 4.4.1]): The Shunkov $2^{\prime}$-group with min $-a b$ is Chernikov. In view of these theorems, $C_{G}(H)$ is Chernikov.

Let $F$ be a subgroup of maximal order among all $X \triangleleft H$, for which $C_{G}(X)$ is non-Chernikov. Take $u \in H \backslash F$ such that $u^{p} \in F$ and also $u F \in Z(H / F)$. Since $\langle u>F \unlhd H$ and also $|<u>F|>|F|$, the $C_{G}(<u>F)$ is Chernikov.

Put $T=\langle u\rangle C_{G}(F)$. If $|\langle u\rangle|=p$, then $u^{p}=1 \in Z(T)$. If $|\langle u\rangle| \neq p$, then $H$ and, at the same time, $F$ are cyclic. Therefore in this case we have: $u^{p} \in F \subseteq Z\left(C_{G}(F)\right)$. Consequently, $\left[u^{p}, T\right]=\left[u^{p},<u>C_{G}(F)\right]=1$, i.e. $u^{p} \in Z(T)$.

In view of S. N. Chernikov's Lemma (see, for instance, [1, Lemma 3.7, p. 151]), $C_{T}(u)=\langle u\rangle$ $\left(C_{T}(u) \cap C_{G}(F)\right)$. Then $\left|C_{T}(u): C_{T}(u) \cap C_{G}(F)\right|<\infty$. Since $C_{T}(u) \cap C_{G}(F) \subseteq C_{G}(<u>F)$ and $C_{G}(\langle u\rangle F)$ is Chernikov (see above), the subgroup $C_{T}(u) \cap C_{G}(F)$ is Chernikov too. Therefore $C_{T}(u)$ is also Chernikov.

Further, it is easy to see: the statement (iii) of Theorem with $T$ in the character of $G$ is not valid. Therefore in view of the assertion (3), $T$ contains some normal subgroup $M$ that does not satisfy $\min -a b$ and has no normal locally finite subgroups $\neq 1$.

Let $K$ be a normal subgroup of $T$, having some abelian non-Chernikov subgroup $B$. In view of Lemma 1.2 [15], $K$ contains some subgroup $L \triangleleft T$ with infinite $B / L \cap B$ and non-Chernikov $L \cap B$. Taking this into account it is easy to see: $M$ has some infinite descending series

$$
M=M_{0} \supset M_{1} \supset M_{2} \supset \ldots \supset M_{\alpha} \supset M_{\alpha+1} \supset \ldots \supset M_{\gamma}=\cap_{\alpha<\gamma} M_{\alpha} \supseteq 1
$$

of normal subgroups of $T$ such that all $M_{\alpha}, \alpha<\gamma$, do not satisfy min - $a b$ and $M_{\gamma}$ satisfies min - ab. In view of mentioned Shunkov's and An. Ostilovskiy's Theorems, $M_{\gamma}$ is Chernikov. So $M_{\gamma}=1$. Further, since $C_{M}(u)$ is Chernikov, for some $\beta$ such that $0<\beta<\gamma$, we have: $C_{M_{\beta}}(u)=1$. Take $v \in M_{\beta} \backslash\{1\}$. Then, because of $u^{p} \in Z(T)$, we have: $u, v \in C_{G}\left(u^{p}\right)$ and $\langle u\rangle \cap\left\langle u^{v}\right\rangle=\left\langle u^{p}\right\rangle$. Since $G$ is Shunkov, $\left\langle<u>,\left\langle u^{v}\right\rangle>\right.$ is a finite $p$-group. So $<\langle u\rangle,\left\langle u^{v}\right\rangle>\cap M_{\beta}$ has some element $\neq 1$ centralizing $u$, which is a contradiction.

Thus (iii) is valid.

(5) If for some element $g \in G$ of prime order and for some infinite normal subgroup $H$ of $G$ we have: $H \cap C_{G}(g)=1$, then (iii) is valid. 
Proof. First, give the following Popov-Sozutov-Shunkov Theorem (see Lemmas 2.7, 5.24 [25], Theorem 5.11 [25], Lemma 5.20 [25]): Let $X=U \lambda\langle v\rangle$ be an infinite group with $|\langle v\rangle|=p$, $C_{X}(v)=\left\langle v>\right.$ and $\left.\mid<v, v^{u}\right\rangle \mid<\infty, u \in U$. Then: $X$ is periodic; all divisible abelian subgroups of $U$ belongs to $Z(U)$; every finite subgroup of $U$, normalized by $v$ belongs to some infinite locally finite subgroup of $U$, normalized by $v$. Further, if for some $u \in U$, all subgroups $U \cap\langle u, f v\rangle$ with $f \in U$ are abelian, then the normal closure $\left\langle u^{X}>\right.$ of $u$ in $X$ is abelian.

Now give some comments. Since $\langle v\rangle$ is obviously a Sylow $p$-subgroup of $\left\langle v, v^{u}\right\rangle$ and $\left\langle v, v^{u}\right\rangle=\left(U \cap\left\langle v, v^{u}\right\rangle\right)\langle v\rangle$, for some $w \in U \cap\left\langle v, v^{u}\right\rangle$ we have: $\langle v\rangle^{u}=\langle v\rangle^{w}$, i.e. $u=w$ and $u \in U \cap\left\langle v, v^{u}\right\rangle$. Obviously, for some $a \in\langle u v\rangle$ and $x \in U \cap\left\langle v, v^{u}\right\rangle$, $|\langle a\rangle|=p$ and $\langle a\rangle=\langle v\rangle^{x}$. So $\langle u v\rangle=\langle v\rangle^{x}$. Thus, $\left.(X \backslash U) \cup\{1\}=\cup_{u \in U}<v^{u}\right\rangle$. Hence follows: for $y, z \in X \backslash U,|<y, z>|<\infty$.

Now return directly to the present assertion (5). Since $G$ is Shunkov, for any $x, y \in G$, we have: $\left|<g^{x}, g^{y}>\right|<\infty$.

If $H$ contains a quasicyclic subgroup, then in view of Popov-Sozutov-Shunkov Theorem above, $Z(H)$ contains all such subgroups. Then every maximal abelian subgroup of $H$ contains a quasicyclic subgroup. Consequently in view of Lemma 1.1 [15], all maximal abelian subgroups of $H$ are Chernikov and so $H$ satisfies min - ab. Therefore in view of Suchkova-Shunkov Theorem mentioned above, $H$ is Chernikov. So in accordance with the assertion (2), the statement (iii) is valid.

Now let $H$ have no quasicyclic subgroups. Take $u, f \in H$. For some $h \in H, f g=g^{h}$ (see comments above). Also $H \cap<g^{h}, g^{h u}>$ is a finite subgroup, normalized by $g^{h}$, and $u \in H \cap<g^{h}, g^{h u}>$ (see comments above). Then $H \cap<u, f g>\subseteq H \cap<g^{h}, g^{h u}>$. Further, in view of Popov-Sozutov-Shunkov Theorem above, $H \cap<g^{h}, g^{h u}>$ belongs to some infinite locally finite subgroup $R$ of $H$, normalized by $g^{h}$. By virtue of J. G. Thompson Theorem [26], $R$ is locally nilpotent. Since $R$ has no quasicyclic subgroups, $R$ is also non-Chernikov. Therefore in view of Lemma 2.2 [15], $R$ is abelian. At the same, $H \cap\langle u, f g\rangle$ is abelian. Consequently, in view of Popov-Sozutov-Shunkov Theorem above, $\left\langle u^{H<g\rangle}\right\rangle$ is abelian. Thus $H$ is the product of normal locally finite subgroups $\left\langle u^{H<g\rangle}\right\rangle$, taking by all $u \in H$. Then in consequence of O. Yu. Shmidt's Theorem, $H$ is locally finite. Therefore (iii) is valid (see (2)).

(6) If for $g \in G$ of prime order the centralizer $C_{G}(g)$ satisfies min-ab, then (iii) is valid.

Proof. Let $C_{G}(g)$ satisfy min - $a b$. In view of Suchkova-Shunkov Theorem, $C_{G}(g)$ is Chernikov. Assume that (iii) is not valid. Let $M$ be such as in (3). Then $M$ has some descending series

$$
M=M_{0} \supset M_{1} \supset M_{2} \supset \ldots \supset M_{\gamma}=\cap_{\alpha<\gamma} M_{\alpha}
$$

such that $M_{\gamma} \triangleleft G$ and $M_{\gamma}$ satisfies min - ab, and for $\alpha<\gamma, M_{\alpha} \triangleleft G$ and $M_{\alpha}$ does not satisfy min $-a b$ (see above the proof of the assertion (4)). In view of Suchkova-Shunkov Theorem [8], $M_{\gamma}$ is Chernikov. Consequently $M_{\gamma}=1$. Therefore because of $C_{G}(g)$ is Chernikov, for some $\beta<\gamma$ we have: $C_{G}(g) \cap M_{\beta}=1$. But then, with regard to (5), (iii) is valid, which is a contradiction.

Remind: the group with a normal abelian subgroup of finite index is called almost abelian.

(7) If for $g \in G$ of prime order the $C_{G}(g)$ is almost abelian, then (iii) is valid.

Proof. First, (iii) is valid, if $C_{G}(g)$ is Chernikov (see (6)). Let $C_{G}(g)$ be almost abelian non-Chernikov and $A$ be its abelian subgroup of finite index. Since $A$ is non-Chernikov, it is a direct product of groups of prime orders (see Lemma $1.1[15]$ ). Therefore, obviously, $A$ has an infinite chain $A_{1} \supset A_{2} \supset \ldots \supset A_{n} \supset A_{n+1} \supset \ldots$ with factors of prime orders. Since $G$ satisfies 
min - $a b \bar{c}$, the set of all complemented in $G$ terms of the chain is infinite. Let $D_{n}$ complements some $A_{n}$ in $G$. Then $A=A_{n} \times\left(A \cap D_{n}\right)$ (by S. N. Chernikov's Lemma). In view of Chunikhin's Lemma (see, for instance, [21, Lemma 1.36]), $\left\langle\left(A \cap D_{n}\right)^{G}\right\rangle \subseteq D_{n}$. Since also $D_{n} \cap C_{G}(g)$ is finite, $\left\langle\left(A \cap D_{n}\right)^{G}>\cap C_{G}(g)\right.$ is finite too. Therefore the centralizer of $g$ in $\langle g\rangle\left\langle\left(A \cap D_{n}\right)^{G}\right\rangle$ is finite. Then in view of the assertion (6), the statement (iii) with $\langle g\rangle<\left(A \cap D_{n}\right)^{G}>$ in the character of $G$ is valid. At the same time, $\left\langle\left(A \cap D_{n}\right)^{G}>\right.$ is locally finite. Then in consequence of O. Yu. Shmidt's Theorem, the product of subgroups $\left\langle\left(A \cap D_{n}\right)^{G}\right\rangle$, taken by all complemented in $G$ subgroups $A_{n}$, is an infinite normal locally finite subgroup of $G$. Therefore in view of assertion (2), the statement (iii) is valid.

(8) For $g \in G$ and $\pi=\pi\left(<g>\right.$ ) and $H=<g^{G}>$, all $\pi^{\prime}$-subgroups of $C_{H}(g)$ are Chernikov.

Proof. Assume that $C_{H}(g)$ has some non-Chernikov $\pi^{\prime}$-subgroup. Then in view of SuchkovaShunkov Theorem (mentioned above), this subgroup has some infinite chain $A \supset A_{1} \supset A_{2} \supset$ $\ldots \supset A_{n} \supset A_{n+1} \supset \ldots$ of abelian subgroups. Some $A_{n}$ has a complement $D$ in $G$. Then, with regard to S.N. Chernikov's Lemma, we have:

$$
A \times<g>=A_{n} \times(D \cap A \times<g>)=A_{n} \times(D \cap A) \times(D \cap<g>)=A \times(D \cap<g>) .
$$

Therefore, clearly, $\langle g\rangle=D \cap\langle g\rangle$, i.e. $\langle g\rangle \subseteq D$. Since also $G=(A \times\langle g\rangle) D$, by virtue of Chunikhin's Lemma (see, for instance, [21, Lemma 1.36]), $H \subseteq D$. But $A \subseteq H$ and $A \nsubseteq D$, which is a contradiction.

(9) If $G$ satisfies min - $p^{\prime}$ for some $p$, then (iii) is valid and also $G$ is Chernikov or contains a normal elementary abelian p-subgroup of finite index.

Proof. Assume that (iii) is not valid. Let $M$ be from the assertion (3). In view of the assertion (4), every $p$-subgroup of $G$ is abelian or Chernikov. Consequently, $M$ has an element $g$ of prime order $q \neq p$. Put $H=<g^{M}>$. In view of the assertion (8), in $C_{H}(g)$ all $q^{\prime}$-subgroup are Chernikov. Consequently $C_{H}(g)$ satisfies $\min -p$. Also $C_{H}(g)$ satisfies $\min -p^{\prime}$.

Further, every abelian subgroup of $C_{H}(g)$ is a direct product of a $p$-subgroup and a $p^{\prime}$ subgroup. Thus it is a direct product of two Artinian subgroups, and so it is Artinian. Thus, $C_{H}(g)$ satisfies $\min -a b$. Then in view of the assertion (6), the statement (iii) with $H$ in the character of $G$ is valid. Therefore $H$ is a normal locally finite subgroup of $M$, which is a contradiction. Thus, (iii) is valid.

Now let $G$ be non-Chernikov. Then, with regard to N. V.Chernikova's Theorem $[9,10]$ (see also Introduction), $G=U \lambda V, U$ and $V$ are abelian, $U$ is a direct product of normal in $G$ subgroups of prime orders and $G$ has no quasicyclic subgroups. So $U=U_{p} \times U_{p^{\prime}}, V=V_{p} \times V_{p^{\prime}}$, where $U_{p}$ and $V_{p}$ are $p$-subgroups, $U_{p^{\prime}}$ and $V_{p^{\prime}}$ are $p^{\prime}$-subgroups. Since $U_{p^{\prime}}$ and $V_{p^{\prime}}$ are Artinian abelian, by Kurosh'es Theorem (see, for instance, [19, Proposition 4.2.11, p. 101]), $U_{p^{\prime}}$ and $V_{p^{\prime}}$ are Chernikov. Since $G$ has no quasicyclic subgroups, $U_{p^{\prime}}$ and $V_{p^{\prime}}$ are finite. Therefore $\left|G: U_{p} \lambda V_{p}\right|<\infty$. Since $U_{p}$ is obviously a direct product of normal in $G$ subgroups of order $p$, if $U_{p} \neq 1$, and $V_{p}$ is a $p$-subgroup, $U_{p} \lambda V_{p}=U_{p} \times V_{p}$. In consequence of Lemma 1.1 [15], $U_{p} \times V_{p}$ is elementary abelian.

(10) The statement (iii) is necessarily valid.

Proof. Assume that (iii) is not valid. Let $M$ be from (3). Further, let $g$ be an element of some prime order $p$ of $M$. Put $H=<g^{M}>$. Then $C_{H}(g)$ satisfies $\min -p^{\prime}$ (see (8)). Therefore in view of the assertion (9) (with $C_{H}(g)$ instead of $G$ ), $C_{H}(g)$ is almost abelian. Therefore by virtue of the assertion (7), the statement (iii) with $H$ in the character of $G$ is valid. At the same time, $H$ is locally finite, which is a contradiction. 
B. Show that (iii) implies (ii).

Put $A^{*}=C_{G}(A)$. In view of S. N. Chernikov's Lemma, $A^{*}=A \lambda\left(A^{*} \cap B\right)$. Then because of $A$ and $A^{*} \cap B$ are abelian, $A^{*}$ is abelian too. Obviously, $A^{*}=C_{G}\left(A^{*}\right)$. Further, clearly, $D \cap A^{*}=1$. Since $C$ is a direct product of groups of prime orders or $C=1$, we have for some subgroup $C^{*} \subseteq C$ : $B=\left(A^{*} \cap B\right) \times\left(D \times C^{*}\right)$. Then $B=D \times\left(A^{*} \cap B\right) \times C^{*}$. So for $B^{*}=D \times C^{*}$ we have: $G=A^{*} B=A^{*} \lambda\left(D \times C^{*}\right)=A^{*} \lambda B^{*}$. Therefore in view of Proposition 2 [27], every infinite abelian subgroup of $G$ is complemented in it.

Of course, (ii) implies (i).

Theorem is proven.

\section{References}

[1] S.N Chernikov, Groups with prescribed properties of the system of subgroups, Nauka, Moscow, 1980 (in Russian).

[2] N.S.Chernikov, S.N.Chernikov. The Kiev period, Algebra and linear inequalities. To the centenary of the birh of Sergei Nikolaevich Chernikov, Inst. math. and mech. Ural Branch Russian Acad. Sci., Yekaterinburg, 2012, 74-170 (in Russian).

[3] N.S.Chernikov, S.N.Chernikov and the theory of groups. The Kiev period, Inst. math. Nat. Acad. Sci. Ukraine, Kyiv, 2012 (Preprint / NAS of Ukraine. Inst. mat., 2012.01) (in Russian).

[4] V.I.Senashov, V.P.Shunkov, Groups with finiteness conditions, Publishing House Sib. Branch Russian Acad. Sci., Novosibirsk, 2001 (in Russian).

[5] A.Yu.Ol'shanskiy, Geometry of defining relations in groups, Nauka, Moscow, 1989 (in Russian).

[6] O.H.Kegel, B.A.F.Wehrfritz, Locally finite groups, North-Holland Publ. Co, AmsterdamLondon, 1973.

[7] V.P.Shunkov, $M_{p}$-groups, Nauka, Moscow, 1990 (in Russian).

[8] N.G. Suchkova, V.P.Shunkov, On groups with the minimal condition for abelian subgroups, Algebra i Logika, 25(1986), no. 4, 445-469 (in Russian).

[9] N.V. Chernikova (Baeva), Completely factorizable groups, Dokl. Akad. Nauk SSSR, 92(1953), no, 5, 877-880 (in Russian).

[10] N.V.Chernikova, Groups with complemented subgroups, Mat. Sb., 39(1956), no. 3, 273-292 (in Russian).

[11] M.I.Kargapolov, Some problems in the theory of nilpotent and solvable groups, Dokl. Akad. Nauk SSSR, 127(1959), no. 6, 1164-1166 (in Russian).

[12] Yu.M. Gorchakov, Primitive factorizable groups, Nauchn. zametki Perm. univ., 17 (1960), no. 2, 15-31 (in Russian).

[13] B.I.Mishchenko, The solvable groups with complemented infinite abelian subgroups, Groups with prescribed properties of subgroups, Inst. mat. AN USSR, Kiev, 1973, 127-161 (in Russian). 
[14] N.S.Chernikov, Groups with minimal conditions for noncomlemented abelian subgroups, Dokl. Akad. Nauk SSSR, 223(1975), no. 4, 797-798 (in Russian).

[15] N.S.Chernikov, $\omega A$-factorizable groups, Some questions of the theory of groups, In-te math. AN USSR, Kiev, 1975, 100-122 (in Russian).

[16] N.S.Chernikov, Locally finite $\omega \sigma A$-factorizable groups, Investigations on the theory of groups, Inst. mat. AN USSR, Kiev, 1976, 63-110 (in Russian).

[17] N.S.Chernikov, Groups with wide systems of complemented abelian subgroups, Algebra and combinatorics: Thes. of Intern. conf. on algebra and combinatorics, dedicated to the 60-th birthday of A. A. Makhnev, Inst. Mat. i Mekhaniki of the Ural Branch RAS, Yekaterinburg, Russia, 2013, 263.

[18] N.S Chernikov, On groups with complemented infinite abelian subgroups, Mat. Zametki, 28(1980), no. 5, 665-674 (in Russian).

[19] D.J.S.Robinson, A course in the theory of groups, Springer, New York-Heidelberg-Berlin, 1982.

[20] D.J.S.Robinson, Finiteness conditions and generalized soluble groups. Pt.1, Springer, Berlin-Heidelberg-New York, 1972.

[21] N.S.Chernikov, Groups which are products of permutable subgroups, Naukova dumka, Kiev, 1987 (in Russian).

[22] B.I.Plotkin, Groups of automorphisms of algebraic systems, Nauka, Moscow, 1966 (in Russian).

[23] V.P.Shunkov, On a class of p-groups, Algebra i Logika, 9(1970), no. 4, 484-496 (in Russian).

[24] A.N.Ostilovskiy, The locally finiteness of some groups with the minimal condition for abelian subgroups, Algebra i Logika, 16(1977), no. 1, 63-73 (in Russian).

[25] A.M.Popov, A.I.Sozutov, V.P Shunkov, Groups with systems of Frobenius subgroups, Krasnoyarsk gos. techn. univ., Krasnoyarsk, 2004 (in Russian).

[26] J.G.Thompson, Finite groups with fixed-point-free automorphisms of prime order, Proc. Nat. Acad. Sci. USA., 49(1959), 578-581.

[27] S.N.Chernikov, Some types of infinite groups with the given system of complemented infinite abelian subgroups, Algebra i Logika, 15(1976), no. 6, 660-683 (in Russian).

\section{Шунковские группы с условием минимальности для недополняемых абелевых погрупп}

Николай С. Черников

\footnotetext{
В настоящей работе мы даем полное исчерпывающее описание указанных шунковских групп.

Ключевые слова: шунковскал, периодическая, локально конечная, вполне факторизуемая, черниковская группа, условия минимальности, дополняемые, абелевы подгруппы.
} 